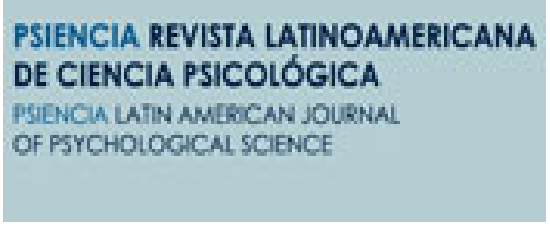

PSIENCIA REVISTA LATINOAMERICANA DE CIENCIA PSICOLOGICA
PSIENCIA. Revista Latinoamericana de Ciencia Psicológica

ISSN: 2250-5490

contacto@psiencia.org

Asociación para el Avance de la Ciencia

Psicológica

Argentina

Viñas Velázquez, Bertha Margarita; Mejía Ramírez, Manuel Alejandro; Martínez Álvarez, Yaroslavna; Islas Limón, Julieta Yadira SEXUALIDAD POSITIVA Y PREVENCIÓN DE ABUSO SEXUAL INFANTIL EN LA FRONTERA NORTE DE MÉXICO

PSIENCIA. Revista Latinoamericana de Ciencia Psicológica, vol. 7, núm. 1, 2015, pp. 916

Asociación para el Avance de la Ciencia Psicológica

Buenos Aires, Argentina

Disponible en: http://www.redalyc.org/articulo.oa?id=333139283002

Cómo citar el artículo

- Número completo

- Más información del artículo

- Página de la revista en redalyc.org 


\section{SEXUALIDAD POSITIVA Y PREVENCIÓN DE ABUSO SEXUAL INFANTIL EN LA FRONTERA NORTE DE MÉXICO}

\author{
POSITIVE SEXUALITY AND PREVENTION OF CHILD SEXUAL ABUSE IN \\ THE NORTHERN BORDER OF MEXICO
}

\author{
Bertha Margarita Viñas Velázquez', Manuel Alejandro Mejía Ramírez,2, \\ Yaroslavna Martínez Álvarez, Julieta Yadira Islas Limón' \\ ${ }^{1}$ Universidad Autónoma de Baja California, \\ ${ }^{2}$ Universidad de las Californias Internacional \\ margarita.vinas@uabc.edu.mx
}

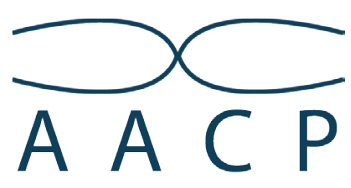

ASOCIACIÓN PARA EL AVANCE DE LA CIENCIA PSICOLÓGICA

doi: $10.5872 /$ psiencia/7.1.22 (c) $2015 \cdot$ www.psiencia.org

Cómo citar este artículo:

Viñas Velázquez, B. M., Mejía Ramírez, M. A., Martínez Álvarez, Y., \& Islas Limón, J. Y. (2015). Sexualidad Positiva y prevención de abuso sexual infantil en la frontera norte de México. PSIENCIA. Revista Latinoamericana de Ciencia Psicológica, 7, 9-16.

Resumen: Se investigó sobre el conocimiento que poseen niños y niñas de primer grado en cuanto a la sexualidad. Se presentan los resultados de un programa de sexualidad para niños de 5 a 7 años de edad, cuyo objetivo fue fomentar una actitud positiva, duradera y natural sobre la sexualidad y desarrollar habilidades de auto-protección. El programa consistió en trece talleres. Se diseñó y se aplicó un cuestionario para evaluar la efectividad del programa, la comparación de puntajes antes y después del programa indica que los niños y niñas aprendieron elementos clave del programa. Se concluye que este tipo de programas de sexualidad es efectivo para informar a los niños y niñas, y se argumenta que esto conlleva a facilitarles la detección de riesgo de abuso sexual y, por lo tanto, reducirlo.

Palabras clave: Educación sexual - Programa de prevención de abuso sexual infantil - Abuso sexual infantil.

Abstract: The knowledge of first grade children regarding sexuality was researched. The results of a sexuality program for children from 5 to 7 years of age are presented, which sought to instill in these children a durable, positive and natural attitude towards sexuality, and to develop self-protection abilities. A questionnaire was designed and applied to assess the program effectiveness by comparing pre- and post-test measurements. Statistically-significant differences were found that indicate that boys and girls learned key elements of the program. It was concluded that this type of sexuality programs informs children, and therefore, it is argued that it makes easier for them to detect the risk of sexual abuse, to reduce it.

Keywords: Sex education - Child abuse prevention program - Child sexual abuse 


\section{INTRODUCCIÓN}

La sexualidad en niños y niñas es un tema del que no se habla en la sociedad, debido a que es generalmente considerado que "no tienen" sexualidad (Cavanagh, 1999), sin embargo, ésta se desarrolla y expresa mediante la curiosidad y la exploración desde preescolar hasta la pubertad, siendo normal y sano su interés por su propio cuerpo, por las diferencias anatómicas y por el acto sexual (Cavanagh, 1999; Honing, 1998). Aunque estas conductas son sanas, pueden ser objeto de preocupación cuando son excesivas o van más allá de los límites impuestos por los padres (Cavanagh, 1999), en cuyo caso, podrían ser un indicador de una problemática más apremiante, como es que haya sido víctima de abuso sexual (Echeburúa \& de Corral, 2006).

El abuso sexual es toda agresión física o psicológica contra la sexualidad del niño, que afecte su bienestar y viole sus derechos para satisfacer los deseos de un adulto (Besten, 2001). Puede o no incluir tocamiento genital o de otro tipo en el cuerpo del menor, que el agresor muestre sus órganos sexuales u obligar al niño a tocarlos, cualquier tipo de penetración o intento de ella con alguna parte del cuerpo o con objetos, realizar o enseñar pornografía al niño o la niña, que pueden ocurrir en un mismo momento o por separado, en una sola ocasión o durante un periodo prolongado de tiempo (Arredondo, 2002), y se puede presentar independientemente del sexo, raza, grupo étnico o clase social (Fuqua, 2008).

El abuso sexual es una realidad preocupante debido a su prevalencia; $9.1 \%$ de las niñas y $2.8 \%$ de los niños en Baja California llegan a sufrir maltrato sexual (Villatoro, Quiroz, Gutiérrez, Díaz \& Amador, 2006). La Secretaría de Salud Pública, en México, reportó en el 2010 haber recibido 59,240 denuncias de maltrato infantil de las cuales se comprobó el maltrato en 34,023 casos y se llevaron ante el ministerio público 4,862 . En el mismo año, en Baja California se recibieron 2,048 denuncias de maltrato infantil, en 272 casos se comprobó el maltrato y 244 fueron presentados ante el ministerio público. México ha visto un incremento de casos de abuso sexual infantil: en 1998 la prevalencia era de $4.3 \%$ en hombres y mujeres (Ramos-Lira, Saldívar-Hernández, Medina-Mora, Rojas-Guiot \& Villatoro-Velázquez, 1998), mientras que una comparación de tres sondeos (2006, 2009 y 2012) en la Ciudad de México mostró un incremento en la prevalencia tanto en hombres como en mujeres, de $5.2 \%$ a $10.3 \%$ (Bustos et al., 2013). La edad promedio de aquellos que han sido abusados es de 5.7 años y en la mayoría de los casos conocían al perpetrador, quien varía en ser un hermano, padrastro, un tío o inclusive un padre, según los hallazgos de la psicóloga Ruth González Serratos, especialista de la Universidad Autónoma de México (Jarquín, 2003).

En Reino Unido, Estados Unidos, Australia y Canadá cada año se reportan de 1.5 al $5 \%$ de la población de niños a agencias de protección por maltrato. En contraste, en encuestas a niños, adolescentes y adultos, se encuentra que el porcentaje de niños que viven cualquier tipo de maltrato es mucho mayor al que se reporta en las agencias de protección (físico, 4 a 16\%; psicológico, 10\%; negligencia, 1 a 15\%; exposición a violencia doméstica, 10 a 20\%) (Gilbert et al., 2009). Esto demuestra la disparidad que hay entre la cifra que se denuncia y la cifra que realmente se presenta, y evidencia la necesidad de prevención a nivel primario para acortar la brecha entre ambas cifras (Topping \& Barron, 2009).

Marchand, Deneyer y Vandenplas (2011) proponen dividir los factores de riesgo en tres áreas: (a) en el menor: dificultades emocionales o conductuales, enfermedad crónica, enfermedad física, trastornos del desarrollo, nacimiento prematuro, y ser producto de un embarazo no deseado, (b) en los padres: baja autoestima, pobre control de impulsos, abuso de sustancias nocivas, ser joven, haber sido abusados en la infancia, depresión, trastornos mentales, bajo conocimiento del desarrollo infantil, padre o madre soltero/a, expectativas poco realistas, y percepción negativa de la conducta normal de un niño, y (c) por factores socioeconómicos, como: el aislamiento social, pobreza, desempleo, poco acceso a la educación, violencia intrafamiliar.

Existen consecuencias del abuso sexual infantil a corto, mediano y largo alcance, que pueden ser emocionales, cognitivas y conductuales (Arredondo, 2002; Montero \& Cerda, 2002), y afectan la manera de desenvolverse de la persona en las distintas áreas de su vida.

La manera más efectiva de disminuir el abuso sexual infantil es la prevención (Guterman, 2001). Diversas investigaciones demuestran la importancia de programas de prevención desde múltiples dimensiones de la vida del niño (Finkelhor, 2009), entre ellos, los programas de prevención aplicados en el medio escolar. 
De acuerdo con Rispens, Aleman y Goudena (1997), los programas de prevención del abuso sexual aplicados en el medio escolar empoderan a los niños con conocimientos y habilidades para su auto-protección. Topping y Barron (2009) revisaron la efectividad de este tipo de programas: analizaron 22 estudios de países de habla inglesa, y concluyeron que estos programas mejoran el nivel de conocimiento de los participantes respecto a la sexualidad y la prevención del abuso sexual; sin embargo, tal efectividad no puede, en sí misma, determinar en qué medida previene el abuso sexual.

Se han señalado desventajas de los programas de prevención del abuso sexual infantil aplicados en el ámbito escolar, entre ellas: a) que este tipo de programas pudieran poner la responsabilidad del abuso en los niños, cuando debe ser algo que corresponde al adulto (Rayner, 1994), b) es difícil que se encuentre intervención clínica accesible para las víctimas de abuso sexual durante la aplicación del programa, puesto que los aplicadores pueden ser docentes que no tengan preparación adecuada en cuanto a información o sensibilidad con estos temas, c) que el programa sea demasiado corto o no sea tratado con la seriedad merecida debido a prioridades curriculares, y d) la falta de evidencia de la efectividad de estos programas en el contexto real y a largo plazo, a causa de las limitaciones metodológicas de los estudios realizados hasta el momento (Topping \& Barron, 2009).

En el presente estudio se toman en consideración estas desventajas. Por ese motivo, fue manejado por personas con formación en psicología y, entrenamiento específico en la aplicación del programa. Topping y Barron (2009) proponen, además, diversos puntos para la superación de las desventajas: (a) evaluar la efectividad del programa; (b) incorporar el repaso de las habilidades, modelamiento y discusión; (c) duración mínima de 4 o 5 horas; (d) ser aplicado por un amplio rango de personal; e (e) inclusión de los padres. A lo que se agrega que el personal que imparta el programa sea sensible y esté capacitado en el desarrollo de la sexualidad.

Un estudio que evaluó la efectividad a largo plazo en la prevención de abuso sexual es un estudio retrospectivo (Gibson \& Leitenberg, 2000) que encontró evidencia de una menor prevalencia de abuso sexual en mujeres universitarias en Estados Unidos, participantes en algún momento en un programa preventivo (8\%) comparada con mujeres que no participaron en ningún programa de este tipo (14\%). Debido a la falta de más estudios, es conveniente continuar con la investigación en esta área.

Çeçen-Eroğul y Hasirci (2013) llevaron a cabo un programa de prevención de abuso sexual infantil en Turquía, donde encontraron dificultades similares a las condiciones de México, como son: falta de investigaciones publicadas, material, programas previamente aplicados y estandarizados. Ellos aplicaron el programa a niños de 4to grado de primaria, con un diseño cuasi-experimental, con pre y post test y grupo control. Los participantes del grupo experimental aumentaron significativamente su conocimiento en los temas, comparados con el grupo control.

El diseño del presente estudio es similar al de Çeçen-Eroğul y Hasirci (2013) por lo que es de esperarse encontrar resultados análogos, aunque difieren en: el grado escolar, y ausencia de grupo control. El presente estudio evaluó la aplicación del programa psico-educativo de sexualidad intitulado "CRECE", con niños y niñas de primer grado de primaria. Para este propósito, se evaluaron los conocimientos sobre sexualidad de los participantes antes, durante y después de la aplicación del programa. Se buscó: (a) identificar si los niños y niñas adquieren una actitud positiva hacia la sexualidad acorde con su edad como efecto de su participación en el programa, (b) evaluar cuáles conocimientos sobre sexualidad se incrementaron en los participantes a partir de la aplicación del programa, e (c) identificar diferencias en el efecto del programa entre niños y niñas participantes.

\section{METODOLOGÍA}

Diseño. Se utilizó un diseño pre-experimental, incluyendo pre test y post test de los grupos que participaron en el programa, sin grupo control.

Muestra. Participaron alumnos de cuatro grupos mixtos de primer grado de primaria, en dos escuelas con alumnado procedente de familias de clase baja a media, durante el periodo de septiembre a noviembre del 2007. En la primera medición (pre-test) se contó con 134 participantes, a partir de la cual se calculó la consistencia interna del cuestionario. Se seleccionaron sólo los casos que también participaron de la segunda medición (post-test), quedando una muestra de 86 participantes: 39 (45\%) mujeres, y 47 (55\%) hombres. Las edades de los participantes se mantuvieron entre 5 y 7 años. 
Tabla 1. Ejes centrales del taller y del cuestionario

\begin{tabular}{lll}
\multicolumn{1}{c}{ Temática } & \multicolumn{1}{c}{ Variables } & $\begin{array}{c}\text { Ítems del } \\
\text { cuestionario }\end{array}$ \\
\hline "Mi ser único" & Sexo, Diferenciación de los cuerpos, Desarrollo y Reproducción & $1,2,3$ y 14 \\
"Derechos de los niños/as" & Protección, Derechos e Higiene & $5,6,7,9$ y 11 \\
"Diferentes formas de & Límites, Aceptación, Intimidad, Afecto y Autoestima & $4,8,10,12$ y 13 \\
expresar afecto" & Lim \\
\hline
\end{tabular}

Procedimiento. Para la aplicación del programa se obtuvo autorización de la escuela y el consentimiento informado de los padres, madres o tutores de los menores. Las facilitadoras fueron 4 mujeres estudiantes de octavo semestre de la Licenciatura en Psicología, quienes recibieron entrenamiento sobre los contenidos del programa. Se tuvieron sesiones de supervisión trabajando estrategias de conducción de grupos de menores y aspectos generales de la sexualidad humana.

Instrumento. Se preparó un cuestionario que midiera el nivel de conocimiento de los niños y niñas sobre los temas que se discutirían en el programa. El cuestionario se administró antes y después del programa, de forma individual para asegurar la comprensión de cada reactivo. EI cuestionario está formado por 14 reactivos formulados con base en los tres ejes centrales de la estructura de los talleres (ver tabla 1). Cada pregunta evalúa un área distinta, y por lo tanto, se esperaba un análisis de consistencia interna bajo $(\alpha=.23, N=134)$.

Las opciones de respuestas al cuestionario eran: Sí, No y No sé. Cada respuesta era representada con una carita que el niño pudiera identificar fácilmente, para asegurar que dieran la respuesta que intentaban dar (ver tabla 2 en Resultados). Las preguntas 1, 2, 3, 8 y 12 se calificaron con un 1 a la opción No y un 0 a las otras dos opciones. El resto de las preguntas se calificó con un 1 a la opción Sí, y un 0 a las opciones restantes. El puntaje mínimo posible era de 0 , y el máximo de 14 puntos. Estas puntuaciones fueron las que se usaron para los análisis posteriores, a excepción de la tabla 2 (en Resultados).

Contenido del programa. El programa se organizó en 13 temas, divididos en tres ejes: "Mi ser único", con temas relacionados a diferencias genitales, las diferencias físicas entre niños y adultos, y el proceso reproductivo; "Los derechos de los niños", con temas respecto a la autoprotección, derechos e higiene; y "Las diferentes formas de expresar afecto", dividido en establecimiento de límites, intimidad, aceptación, afecto y autoestima. Los temas se organizaron en 13 talleres, que se llevaron a cabo dos veces a la semana en sesiones de 60 minutos. El programa completo duró siete semanas, de septiembre a noviembre de 2007.

\section{RESULTADOS}

Los resultados de las mediciones pre-test y posttest se analizaron con SPSS, y las gráficas se crearon con ggplot2 (Wickham, 2009) en R (R Core Team, 2013). En la tabla 2 se muestran los porcentajes de las respuestas sin calificar de los niños y niñas en el pre-test y el post-test. En todas las preguntas, excepto 9 y 11 , hubo un incremento en el porcentaje de niños que eligió la respuesta esperada. En las preguntas 9 y 11 , el $100 \%$ de los niños incluidos en el análisis respondieron "Si" en el pre-test, y un bajo porcentaje (2.33\% y $1.16 \%$, respectivamente) cambió su respuesta a "No" en el post-test.

Se analizaron los puntajes del cuestionario utilizando un diseño ANOVA mixto de dos factores: sexo (niños y niñas) y tiempo de la medición (pretest, post-test), incluyendo la interacción entre ambos. Se asumieron varianzas iguales entre los dos grupos (niños y niñas) en la condición pre-test $[F$ $(1,84)=0.54, p=.464]$ y post-test $[F(1,84)=1.80$, $p=.183]$ a partir de la prueba de Levene. Las diferencias entre los puntajes del pre-test y del posttest se aproximaron a una distribución normal. Niños y niñas, en conjunto, obtuvieron puntajes mayores en la condición post-test comparada con la condición pre-test $[F(1,84)=66.81, p<.001]$ (ver tabla 3 ), con un efecto medio $\left(\eta^{2}=0.443\right)$, mientras que no se encontraron diferencias entre niños y niñas $\left[F(1,84)=0.67, p=.415, \eta^{2}=0.008\right]$, y tampoco se observó interacción entre las dos va- 
Tabla 2. Porcentaje de respuestas a las preguntas del cuestionario.

\begin{tabular}{|c|c|c|c|c|c|c|}
\hline \multirow{3}{*}{ Preguntas } & \multicolumn{6}{|c|}{ Respuestas (\%) } \\
\hline & \multicolumn{2}{|c|}{ Sí } & \multicolumn{2}{|c|}{ No } & \multicolumn{2}{|c|}{ No sé } \\
\hline & Pre & Post & Pre & Post & Pre & Post \\
\hline 1. Los niños y las niñas somos iguales & 18.60 & 9.30 & 77.91 & 90.70 & 3.49 & 0 \\
\hline $\begin{array}{l}\text { 2. Los niños y las niñas hacemos de la misma manera de } \\
\text { la pipí }\end{array}$ & 8.14 & 5.81 & 89.53 & 91.86 & 2.33 & 2.33 \\
\hline 3. Mi cuerpo es igual al de un adulto & 23.26 & 8.14 & 73.26 & 89.53 & 3.49 & 2.33 \\
\hline 4. Todos los niños y niñas son únicos & 44.19 & 69.77 & 40.70 & 22.09 & 15.12 & 8.14 \\
\hline 5. Nuestro cuerpo es importante & 91.86 & 98.84 & 6.98 & 1.16 & 1.16 & 0 \\
\hline 6. Uno de mis derechos es tener nombre y apellido & 88.37 & 97.67 & 3.49 & 0 & 8.14 & 2.34 \\
\hline 7. Es importante que me bañe & 98.84 & 100 & 0 & 0 & 1.16 & 0 \\
\hline 8. Un desconocido me puede abrazar & 17.44 & 6.98 & 81.40 & 91.86 & 1.16 & 1.16 \\
\hline 9. Es importante que me lave los dientes & 100 & 97.67 & 0 & 2.33 & 0 & 0 \\
\hline 10. Solo mi mama me puede ver sin ropa & 59.30 & 81.40 & 33.72 & 16.28 & 6.98 & 2.33 \\
\hline 11. Tengo derecho de ir a la escuela & 100 & 98.84 & 0 & 1.16 & 0 & 0 \\
\hline 12. Mi cuerpo siente igual si lo acarician o lo pellizcan & 23.26 & 4.65 & 68.60 & 93.02 & 6.98 & 2.33 \\
\hline 13. Todos los niños y niñas son especiales & 82.56 & 94.19 & 10.47 & 2.33 & 6.98 & 3.49 \\
\hline 14. Los bebés nacen de la panza de las mamás & 97.67 & 100 & 1.16 & 0 & 1.16 & 0 \\
\hline
\end{tabular}

Nota: $N=86$

riables $\left[F(1,84)=0.03, p=.858, \eta^{2}<0.001\right]$. Esto indica que niños y niñas obtuvieron en promedio puntajes mayores después de haber participado en el programa, sin que el programa tuviera un efecto diferenciado hacia alguno de los dos sexos.

Al compararse los tres grupos de preguntas a partir de los tres temas globales del programa (tabla 1 en Metodología), se obtuvieron diferencias significativas en los tres usando la prueba de rangos de Wilcoxon $(p<.001, p=.023$ y $p<.001$, respectivamente).
Se aplicó el procedimiento de la prueba de McNemar (para muestras relacionadas) para analizar cada pregunta, con la cual se comparan solamente los casos de participantes que cambiaron su respuesta entre el pre y el post test. Se obtuvieron diferencias significativas sólo en las preguntas $1(p=.027), 3(p=.007), 4(p<.001), 6(p=$ $.039), 8(p=.035), 10(p=.001), 12(p<.001), y$ $13(p=.021)$. Es interesante notar que, para las preguntas que sí mostraron diferencias significativas, el promedio del porcentaje de niños y niñas

Tabla 3. Comparación de puntajes en el cuestionario entre niños y niñas, en la medición pre-test y post-test.

\begin{tabular}{lcccccc} 
& Sexo & Media & $\boldsymbol{D E}$ & $\begin{array}{c}\text { Error } \\
\text { estándar }\end{array}$ & $\boldsymbol{N}$ & $\begin{array}{c}\text { Intervalo de confianza (IC) } \\
\mathbf{9 5 \%}\end{array}$ \\
\hline \multirow{3}{*}{ Pre-test } & Niñas & 11.44 & 1.635 & 0.249 & 39 & {$[10.94-11.93]$} \\
& Niños & 11.62 & 1.483 & 0.227 & 47 & {$[11.17-12.07]$} \\
& Total & 11.53 & 1.547 & 0.168 & 86 & {$[11.19-11.86]$} \\
\hline \multirow{3}{*}{ Post-test } & Niñas & 12.82 & 1335 & 0.210 & 39 & {$[12.40-13.24]$} \\
& Niños & 13.06 & 1.292 & 0.191 & 47 & {$[12.68-13.44]$} \\
& Total & 12.94 & 1.310 & 0.142 & 86 & {$[12.66-13.23]$} \\
\hline
\end{tabular}


que contestaron correctamente en el pre-test fue de $71.9 \%$; en cambio, el promedio de aciertos en el pre-test fue de $96.3 \%$ para las preguntas que no mostraron diferencias significativas. Es decir, el efecto del programa se observó en aquellas preguntas que en el pre-test obtuvieron más bajos índices de respuestas correctas, y no así en las preguntas que obtuvieron puntajes altos. La figura 1 muestra el punto anterior de una manera más clara: las preguntas con menor puntaje en el pre-test, que a su vez se concentraron en el tema de diferentes formas de expresar afecto (ej. preguntas 4,10 , y 12, con cambios de 25.58, 22.1 y 24.42 puntos porcentuales, respectivamente, en un sentido positivo), fueron las que en mayor pro- porción obtuvieron incrementos significativos en el post-test. Esto podría indicar que el taller fue efectivo en los temas que requerían mayor trabajo, a comparación de temas que no parecían requerir mayor intervención.

Durante el desarrollo del programa, fue notorio el cambio de actitud de los menores en forma positiva, variando su percepción y respuesta. Por ejemplo, en el establecimiento de límites, redirigiéndolos a su protección y al de sus compañeros, sobresale una de las sesiones donde a través de títeres se representó un niño lastimado del brazo y el proceso de estar en clases en esa situación; al principio jugaban al respecto, poco a poco

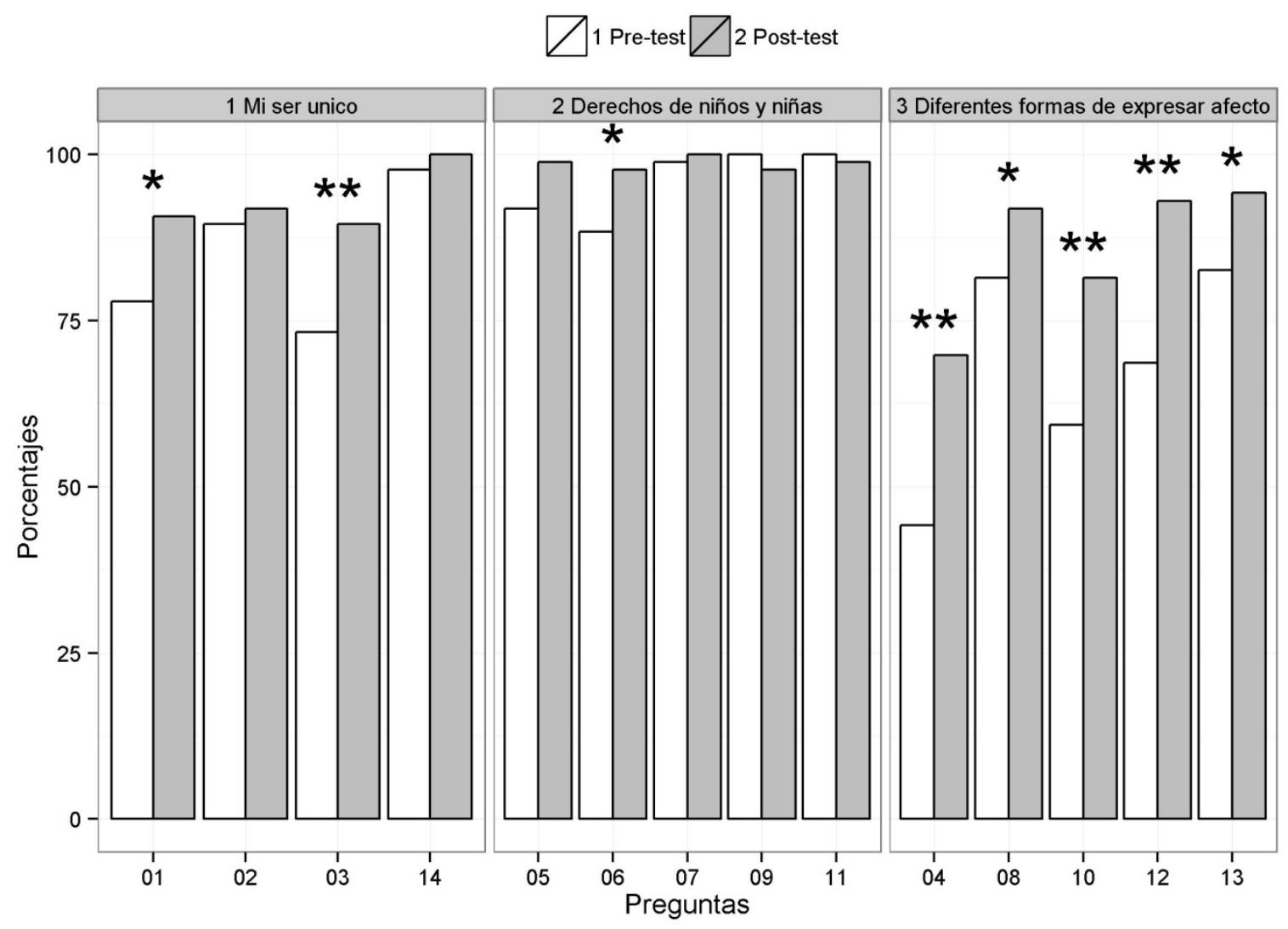

Figura 1. Porcentajes de niños y niñas que dieron la respuesta esperada en el pre-test y en el post-test.

Nota: Las preguntas están separadas en los tres ejes del cuestionario. Se observa que las preguntas con menor puntaje en el pretest tuvieron mayor cambio en el post-test, y estos cambios fueron estadísticamente significativos (ej. preguntas 3, 4, 10, y 12), en cambio, las preguntas con mayor puntaje en el pre-test no obtuvieron cambios significativos (ej. preguntas $07,09,11, \mathrm{y} 14$ ). ${ }^{*} p<.05$, ${ }^{* *} p<.01$. 
preguntaron su sentir y mostraron preocupación por protegerle, no dañarle e integrarlo con ellos, y mostraron empatía al externar alguna situación similar que ellos hubieron pasado.

Por otra parte, se logró que reconocieran a niños y niñas como dos entidades distintas en base a sus diferencias fisiológicas y no por papeles sociales, forma de vestir o longitud de cabello. Lo que al principio fue causa de risa o juego, durante el transcurso de las sesiones se convirtió en temas comunes sin que surgiera morbo; así, cuando se llegó al tema de reproducción sexual y expresión de lo que su cuerpo sentía, fue fácil la formación de equipos heterogéneos, así como la participación activa de cada niño y niña de forma aleatoria, en un marco de respeto a lo que otros manifestaban.

\section{DISCUSIÓN}

El programa incrementó los conocimientos sobre sexualidad en los niños y niñas que participaron, sin tener un efecto diferenciado entre niños o niñas. Sumado a eso, los participantes adquirieron una actitud positiva hacia la sexualidad acorde con su edad como efecto de su participación en el programa. Los resultados se asemejan a los encontrados por Çeçen-Eroğul y Hasirci (2013), sobre un programa de prevención de abuso sexual con niños de $4^{\circ}$ grado en Turquía. Al igual que en dicho estudio, el presente cuenta con la fortaleza de probar que el trabajar estos temas con niños y niñas a nivel primaria mejora notablemente su conocimiento en áreas relacionadas con la sexualidad, y esto, como consecuencia, podría prevenir conductas que pongan a los menores en riesgo de abuso sexual. Sin embargo, la debilidad de este tipo de estudio recae en la incapacidad de comprobar si realmente el aprendizaje de estos niños previene efectivamente el abuso sexual.

En línea con los programas de prevención del abuso sexual aplicado en el ámbito escolar, argumentamos que a partir de los resultados de la aplicación del programa de prevención, se espera que los conocimientos efectivamente adquiridos por los menores sean suficientes para alejarlos de conductas que los expongan a situaciones de riesgo, previniendo así el abuso sexual. Con esto no se pretende insinuar que la responsabilidad del riesgo del abuso sexual cae sobre el menor, pero se cree que el conocimiento adecuado de la sexualidad a esta edad los empodera para evitar dichas situaciones, o para comunicar de una manera sana a los adultos cuando se sienten amenazados o han sido abusados. Sería necesario un seguimiento a este estudio para obtener información acerca de la conexión entre los conocimientos y habilidades promovidos por este programa y el riesgo de abuso sexual, y esto se podría lograr al aplicar un cuestionario al alumnado que participó en este programa en futuros años.

\section{CONCLUSIÓN}

El aprendizaje de los niños y niñas, a partir de haber recibido el programa de sexualidad saludable, fue significativo, tanto en la adquisición de conocimientos como en la adopción de una actitud positiva hacia el tema. El eje de mayor aprendizaje fue el de diferentes formas de expresar afecto, posiblemente debido a que era la temática donde menores puntajes se observaron en el pre-test. El programa posiblemente funcionó como reforzador en los otros dos ejes. La participación de los niños en el programa les ayuda a formar una percepción positiva respecto a la sexualidad desde temprana edad. Puede considerarse necesario comenzar desde edades más tempranas, es decir, a nivel preescolar. La debilidad del estudio recae en su corto alcance para comprobar la influencia del conocimiento adquirido por los menores sobre el riesgo de abuso sexual. Se exhorta a futuros investigadores, que se interesen en la investigación y prevención del abuso sexual infantil, a buscar maneras efectivas de comprobar el beneficio de este tipo de programas en la prevención del abuso sexual infantil.

\section{REFERENCIAS}

Arredondo Ossandon, V. (2002). Guía Básica de Prevención del Abuso Infantil. Corporación de Promoción de Apoyo a la Infancia. Chile: Paicabí.

Besten, B. (2001). Abusos sexuales en los niños ( $2^{\mathrm{a}}$ Ed.). Barcelona, España: Herder.

Bustos, M., Oliva, N., Fleiz, C., Fregoso, D., Moreno, M., Mujica, A., ... Medina-Mora, M. E. (2013). Abuso sexual en hombres y mujeres. Tendencias que no reflejan diferencias: Resumen Ejecutivo. México, D.F.: INPRFM, IAPA \& AFSEDF.

Cavanagh Johnson, T. (1999). Understanding Your Child's Sexual Behavior: What's Natural 
and Healthy. Oakland, California: New Harbinger Publications.

Çeçen-Eroğul, A., \& Hasirci, Ö. (2013). The Effectiveness of Psycho-educational School-based Child Sexual Abuse Prevention Training Program on Turkish Elementary Students. Educational Sciences: Theory \& Practice, 13, 725729.

Echeburúa, E., \& De Corral, P. (2006). Secuelas emocionales en víctimas de abuso sexual en la infancia. Cuadernos de Medicina Forense, 12, 75-82.

Finkelhor, D. (2009). The Prevention of Childhood Sexual Abuse. Future Of Children, 19, 169-194.

Fuqua, D. S. (2008). Safe @ Last: The Evaluation of a Child Sexual Abuse Prevention Program for Elementary Students. Michigan, Estados Unidos: ProQuest \& UMI Dissertation Publishing.

Gibson, L. E., \& Leitenberg, H. (2000). Child Sexual Abuse Prevention Programs: Do They Decrease the Occurrence of Child Sexual Abuse?. Child Abuse \& Neglect, 24, 1115-25.

Gilbert, R., Widom, C. S., Browne, K., Fergusson, D., Webb, E., \& Janson, S., (2009). Burden and Consequences of Child Maltreatment in HighIncome Countries. The Lancet, 373, 68-81. doi: 10.1016/S0140-6736(08)61706-7

Guterman, N. (2001). Stopping child maltreatment before it starts. California, Estados Unidos: Sage.

Jarquín, E. (2003). Alarmantes cifras de abuso sexual infantil en México. CIMAC Noticias. Disponible en: http://cimacnoticias.com.mx/ node/28195

Marchand, J., Deneyer, M., \& Vandenplas, Y. (2012). Detection, Diagnosis, and Prevention of Child Abuse: the Role of the Pediatrician.
European Journal Of Pediatrics, 171, 17-23.

Montero, A., \& Cerda, C. (2002). Principales necesidades de atención de las víctimas de delitos sexuales. Revista de la Sociedad Chilena de Obstetricia y Ginecología Infantil y de la Adolescencia, 9, 31-36.

Ramos-Lira, L., Saldívar-Hernández, G., MedinaMora, M. E., Rojas-Guiot, E., \& Villatoro-Velázquez, J. (1998). Prevalencia de abuso sexual en estudiantes y su relación con el consumo de drogas. Salud Pública, 40, 221-233.

R Core Team (2013). R: A Language and Environment for Statistical Computing. Vienna, Austria: R Foundation for Statistical Computing.

Rayner, M. (1994). The Commonwealth's Role in Preventing Child Abuse: A Report to the Minister for Family Services. Melbourne: Australian Institute for Family Studies.

Rispens, J., Aleman, A., \& Goudena, P. P. (1997). Prevention of child sexual abuse victimization: A Meta-Analysis of School Programs. Child Abuse and Neglect, 21, 975-987.

Secretaría de Salud Pública (2010). Maltrato y abuso infantil en México: factor de riesgo en la comisión de delitos. México: Autor.

Topping, K. G, \& Barron, I. G. (2009). School-Based Child Sexual Abuse Prevention Programs: A Review of Effectiveness. Review Of Educational Research, 79, 431.

Villatoro, J., Quiroz, N., Gutiérrez, M. L., Díaz, M., \& Amador, N. (2006). ¿Cómo educamos a nuestros/as hijos/as? Encuesta de Maltrato Infantil y Factores Asociados 2006. México: INMUJERES \& Instituto Nacional de Psiquiatría.

Wickham, H. (2009). ggplot2: Elegant Graphics for Data Analysis. Nueva York, Estados Unidos: Springer.
PSIENCIA. REVISTA LATINOAMERICANA DE CIENCIA PSICOLÓGICA PSIENCIA. LATIN AMERICAN JOURNAL OF PSYCHOLOGICAL SCIENCE 